\title{
Entropy Generation in MHD Free Convective Boundary Layer Flow Past an Inclined Flat Plate Embedded in a Porous Medium with Hall Currents
}

\author{
S. Das \\ Department of Mathematics \\ University of Gour Banga \\ Malda 732 103, India
}

\author{
B. C. Sarkar \\ Department of \\ Applied Mathematics \\ Vidyasagar University \\ Midnapore 721 102, India
}

\author{
R. N. Jana \\ Department of \\ Applied Mathematics \\ Vidyasagar University \\ Midnapore 721 102, India
}

\begin{abstract}
An analysis of entropy generation in an MHD boundary layer flow of a viscous incompressible electrically conducting fluid past an inclined flat plate embedded in a porous media in a rotating system with Hall currents has been presented. The governing equations describing the flow have been solved analytically. The velocity field, induced magnetic field, shear stress and bulk temperature in the boundary layer flow have been discussed with the help of graphs. The entropy generation is estimated via an analytical solution of the temperature and velocity profiles obtained from the momentum and energy equations governing the flow. The Bejan number is also obtained and discussed.
\end{abstract}

Keywords: MHD boundary layer flow, Hall currents, Grashof number, rotation parameter, angle of inclination, bulk temperature, entropy generation rate, Bejan number and porous medium.

\section{INTRODUCTION}

Analysis of hydromagnetic boundary layer flow and heat transfer of electrically conducting fluids is of great interest in many branches of engineering. Practical applications are found in the design of cooling systems for electronic devices, in the field of solar energy collection, geothermal reservoirs, heat exchangers, cooling of an infinite metallic plate in a cooling bath, magnetohydrodynamic (MHD) stirring of molten metal, magnetic-levitation casting, MHD marine propulsion, the boundary layer along a liquid film in condensation processes and a polymer sheet or filament extruded continuously from a dye. MHD flow past a flat surface has many important technological and industrial applications such as micro MHD pumps, micromixing of physiological samples, biological transportation and drug delivery. The application of the magnetic field produces Lorentz forces which are able to transport liquids in the mixing processes as an active micromixing technology method.

In an ionized gas where the density is low and/or the magnetic field is very strong, the conductivity normal to the magnetic field is reduced due to the free spiraling of electrons and ions about the magnetic lines of force before suffering collisions and a current is induced in a direction normal to both the electric and magnetic fields. This phenomenon, well known in the literature, is called the Hall effect. The study of magnetohydrodynamic viscous flows with Hall currents has important engineering applications in problems of magnetohydrodynamic generators, Hall accelerators as well as in flight magnetohydrodynamics. The magnetohydrodynamic natural convective boundary layer flow of an electrically conducting fluid along an inclined flat plate is of considerable interest in the technical field due to its frequent occurrence in industrial and technological applications. A new approach on an MHD natural convective boundary layer flow past a flat plate of finite dimensions has been studied by Ghosh and Pop [1]. Katagiri [2] has investigated the effect of Hall currents on the magnetohydrodynamic boundary layer flow past a semi-infinite flat plate. Pop and Watanabe [3] have discussed the Hall effects on the magnetohydrodynamic boundary layer flow over a continuous moving flat plate. Natural convection heat transfer from a plate with arbitrary inclination has been studied by Fujii and Imura [4]. An analysis of laminar free convective flow and heat transfer about an inclined isothermal plate has been described by Kierkus [5]. Ganesan and Palani [6] have studied the natural convective effects on impulsively started inclined plate with heat and mass transfer. Buoyancy effects in the boundary layers on the inclined, continues, moving sheets have been investigated by Moutsoglou and Chen [7]. Lewandowski [8] has presented the natural convection heat transfer from the plates of finite dimension. He has carried out a theoretical analysis of a hydrodynamic natural convective boundary layer flow with reference to an experimental observation of a double deck boundary layer to an isothermal flat plate.

In these studies, the effects of Darcian fluid flow of a porous medium do not appear in the literature. There is a real background of a heat-absorbing medium in response to a Darcian fluid flow in aerospace science and cryogenics. However, several studies on porous medium flow have been taken into account in past decades. Authors have concentrated to the recent literature on the development of a real-life situation of a practical field of interest. The second law of thermodynamics is applied to investigate the irreversibility in terms of the entropy generation rate. Since entropy generation is the measure of the destruction of the available work of the system, the determination of the factors responsible for the entropy generation is also important in upgrading the system performances. The method is introduced by Bejan $[9,10]$. The entropy generation is encountered in many energy-related applications, such as solar power collectors, geothermal energy systems and the cooling of modern electronic systems. Efficient utilization of energy is the primary objective in the design of any thermodynamic system. This can be achieved by minimizing entropy generation in processes. The theoretical method of entropy generation has been used in the specialized literature to treat external and internal irreversibilities. The irreversibility phenomena, which are expressed by entropy generation in a given system, are related to heat and mass transfers, viscous dissipation, magnetic field etc. Several researchers have discussed the irreversibility in a system under various flow configurations [11-23]. They showed that the pertinent flow parameters might be chosen in order to minimize entropy generation inside the system. Chen and Chen [24] have 
studied the problem of free convective flow of the nonNewtonian fluids past an isothermal vertical flat plate embedded in a porous medium. The hydromagnetic free convective flow with induced magnetic field has been discussed by Ghosh et al.[25]. The natural convection boundary layer flow past a flat plate of finite dimensions embedded in a porous medium has been investigated by Jana et al. [26]. The transient MHD free convection past an infinite vertical porous plate in a rotating system with mass transfer and Hall current has been studied by Ahmed and Kalita [27]. The transient magnetohydrodynamic flow of a visco-elastic fluid past an infinite vertical porous plate embedded in a porous medium with Hall currents and slip condition in a rotating system has been discussed by Ahmed and Talukdar [28]. Das et al. [29] have studied the Hall effects on an MHD free convective boundary layer flow past a vertical flat plate. Makinde [30] has made an analysis on MHD boundary-layer flow and mass transfer past a vertical plate in a porous medium with constant heat flux. Kumar and Chand [31] have studied the effect of slip conditions and Hall currents on an unsteady MHD flow of a visco-elastic fluid past an infinite vertical porous plate through porous medium. Entropy analysis for MHD boundary layer flow and heat transfer over a flat plate with a convective surface boundary condition has been presented by Makinde [32].

The objective of this study is to investigate the entropy generation in magnetohydrodynamic free convection flow past an inclined flat plate embedded in a porous medium in the presence of a uniform transverse magnetic field on taking Hall currents into account. The effects of governing parameters on the flow field and entropy generation are presented graphically and in tabuler form.

\section{MATHEMATICAL FORMULATION AND ITS SOLUTION}

Consider a steady MHD free convective boundary layer flow past a flat plate of finite dimensions of a viscous incompressible electrically conducting fluid confined to the arbitrary inclination $\alpha$ of the plate embedded in a porous medium. A uniform magnetic field of strength $B_{0}$ is imposed perpendicular to the plate. The cartesian coordinates system is chosen in such a way that the $x$ - and $z$-axes are in the plane of the plate and the $y$ axis normal to the plane of the plate (See Fig.1). The fluid and the plate rotate in unison with a uniform angular velocity $\Omega^{*}$ about the $z$-axis normal to the plate. Since the plate is infinitely long all physical quantities, except pressure, will be function of $y$ only. The flow is generated due to the buoyancy force in the presence of a transverse magnetic field.

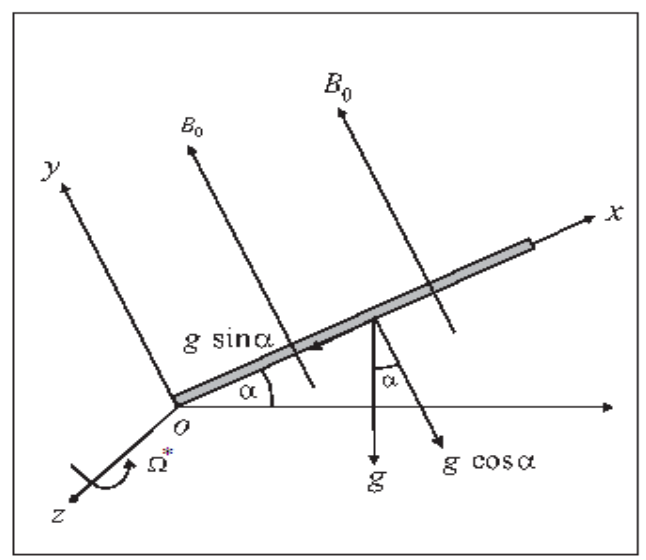

Fig.1: Geometry of the Problem
The equation of continuity $\nabla \cdot \vec{q}=0$ and the no-slip condition at the plate give $v=0$ everywhere in the flow where $\vec{q} \equiv(u, v, w)$ is the fluid velocity. The solenoidal equation $\nabla \cdot \vec{B}=0$ gives $B_{y}=$ constant $=B_{0}$ everywhere in the flow, where $\vec{B} \equiv\left(B_{x}, B_{y}, B_{z}\right)$ is the magnetic field intensity.

Under the usual Boussinesq approximation, the Navier-Stokes equations for the steady flow along the co-ordinate axes are

$$
\begin{aligned}
2 \Omega^{*} w & =v \frac{d^{2} u}{d y^{2}}+\frac{B_{0}}{\rho \mu_{e}} \frac{d B_{x}}{d y}+g \beta\left(T-T_{\infty}\right) \sin \alpha-\frac{\mu^{\prime}}{\rho k^{*}} u, \\
0 & =-\frac{1}{\rho} \frac{\partial p}{\partial y}-\frac{1}{2 \rho \mu_{e}}\left(\frac{d B_{x}^{2}}{d y}+\frac{d B_{z}^{2}}{d y}\right)+g \beta\left(T-T_{\infty}\right) \cos \alpha, \\
-2 \Omega^{*} u & =v \frac{d^{2} w}{d y^{2}}+\frac{B_{0}}{\rho \mu_{e}} \frac{d B_{z}}{d y}-\frac{\mu^{\prime}}{\rho k^{*}} w,
\end{aligned}
$$

where $g$ is the acceleration due to the gravity, $\mu_{e}$ the magnetic permeability, $v$ the kinematic viscosity, $p$ the fluid pressure, $\rho$ the fluid density, $T$ the fluid temperature, $T_{\infty}$ the ambient temperature, $\alpha$ the angle of inclination of the plate, $\beta$ the coefficient of thermal expansion, $\mu^{\prime}$ the coefficient of viscosity of the porous medium and $k^{*}$ the the specific permeability of the medium.

Maxwell's equations in the steady state are

$$
\nabla \times \vec{B}=\mu_{e} \vec{J}, \nabla \times \vec{E}=\overrightarrow{0}, \nabla \cdot \vec{B}=0, \nabla \cdot \vec{D}=\rho_{e},
$$

where $\vec{E}$ is the the electric field vector, $\vec{J}$ the current density vector, $\vec{D}$ the displacement current vector and $\rho_{e}$ the free charge density.

The generalized Ohm's law on taking Hall currents into account and neglecting the ion-slip and thermoelectric effects as well as the electron pressure gradient (see Cowling [33]) for a conducting fluid is

$$
\vec{J}+\frac{\omega_{e} \tau_{e}}{B_{0}}(\vec{J} \times \vec{B})=\sigma[\vec{E}+\vec{q} \times \vec{B}],
$$

where $\omega_{e}, \tau_{e}$ and $\sigma$ denote the cyclotron frequency, the electron collision time and the electrical conductivity of the fluid respectively.

Eliminating $\vec{E}$ from equations (4) and (5), we have the $x$ - and $z$-components of the magnetic induction equation as

$$
\begin{aligned}
& -\frac{d^{2} B_{x}}{d y^{2}}+m \frac{d^{2} B_{z}}{d y^{2}}=\sigma \mu_{e} B_{0} \frac{d u}{d y}, \\
& -\frac{d^{2} B_{z}}{d y^{2}}-m \frac{d^{2} B_{x}}{d y^{2}}=\sigma \mu_{e} B_{0} \frac{d w}{d y},
\end{aligned}
$$

where $m=\omega_{e} \tau_{e}$ is the Hall parameter

We assume the temperature distribution in the boundary layer, according to Lewandowski [8] as

$$
\theta=\frac{T-T_{\infty}}{T_{w}-T_{\infty}}=\left(1-\frac{y}{\delta}\right)^{2},
$$

where $\delta$ is the thickness of the boundary layer, $T_{w}$ the constant wall temperature.

The pressure distribution in the boundary layer is obtained on integration of the equation (2) and using the boundary condition $\left(p=p_{\infty}\right.$ at $\left.y=\delta\right)$ as

$$
\begin{aligned}
p & =p_{\infty}+\rho g \beta\left(T_{w}-T_{\infty}\right)\left(y-\frac{y^{2}}{\delta}+\frac{y^{3}}{3 \delta^{2}}-\frac{\delta}{3}\right) \cos \alpha \\
& -\frac{1}{2 \mu_{e}}\left(B_{x}^{2}+B_{z}^{2}\right) .
\end{aligned}
$$


Differentiating the equation (9) with respect to $x$, we get

$$
\frac{\partial p}{\partial x}=\rho g \beta\left(T_{w}-T_{\infty}\right)\left(\frac{y^{2}}{\delta^{2}}-\frac{2}{3} \frac{y^{3}}{\delta^{3}}-\frac{1}{3}\right) \frac{\partial \delta}{\partial x} \cos \alpha .
$$

According to Fujii and Imura [4] , we assume

$$
\frac{\partial \delta}{\partial x} \cong \frac{\overline{\partial \delta}}{\partial x}=F \cong \text { constant }
$$

where bar denotes the mean value.

The boundary conditions for the velocities, the magnetic fields and the temperature distribution are

$$
\begin{aligned}
& u=0, w=0, B_{x}=0, B_{z}=0 \text { and } T=T_{w} \text { at } \quad y=0, \\
& u=0, w=0, B_{x}=0, B_{z}=0 \text { and } T=T_{0} \quad \text { at } \quad y=\delta .
\end{aligned}
$$

Introducing the non-dimensional variables

$$
\eta=\frac{y}{\delta},\left(u_{1}, w_{1}\right)=\frac{(u, w) \delta}{v},\left(b_{x}, b_{z}\right)=\frac{\left(B_{x}, B_{z}\right)}{\sigma \mu_{e} v B_{0}},
$$

equations (1), (3), (6) and (7) become

$$
\begin{aligned}
& \frac{d^{2} u_{1}}{d \eta^{2}}+M^{2} \frac{d b_{x}}{d \eta}-2 K^{2} w_{1}-\frac{u_{1}}{D a} \\
&=-G r\left[\left(\eta^{2}-\frac{2}{3} \eta-\frac{1}{3}\right) F \cos \alpha-(1-\eta)^{2} \sin \alpha\right] \\
& \frac{d^{2} w_{1}}{d \eta^{2}}+M^{2} \frac{d b_{z}}{d \eta}-2 K^{2} u_{1}-\frac{w_{1}}{D a}=0 \\
& m \frac{d^{2} b_{z}}{d \eta^{2}}-\frac{d^{2} b_{x}}{d \eta^{2}}=\frac{d u_{1}}{d \eta} \\
&-m \frac{d^{2} b_{x}}{d \eta^{2}}-\frac{d^{2} b_{z}}{d \eta^{2}}=\frac{d w_{1}}{d \eta}
\end{aligned}
$$

where $M=\left(\frac{\sigma}{\rho v}\right)^{\frac{1}{2}} B_{0} \delta$ is the Hartmann number, $D a=\frac{k^{*}}{\delta^{2}}$ the Darcy number, $K^{2}=\frac{\Omega^{*} \delta^{2}}{v}$ the rotation parameter and $G r=\frac{g \beta \delta^{3}\left(T_{w}-T_{0}\right)}{v^{2}}$ the Grashof number.

Combining equations (14) and (15) and equations (16) and (17), we get

$$
\begin{aligned}
\frac{d^{2} q}{d \eta^{2}} & +M^{2} \frac{d b}{d \eta}-\left(\frac{1}{D a}+2 i K^{2}\right) q \\
& =-G r\left[\left(\eta^{2}-\frac{2}{3} \eta-\frac{1}{3}\right) F \cos \alpha-(1-\eta)^{2} \sin \alpha\right], \\
\frac{d^{2} b}{d \eta^{2}} & +\frac{1}{1+i m} \frac{d q}{d \eta}=0,
\end{aligned}
$$

where

$$
q=u_{1}+i w_{1}, b=b_{x}+i b_{z} \text { and } i=\sqrt{-1} .
$$

On the use of (13), the boundary conditions for $q(\eta), b(\eta)$ and $\theta(\eta)$ are

$$
\begin{aligned}
& q=0, b=0, \theta=1 \text { at } \eta=0, \\
& q=0, b=0, \theta=0 \text { at } \eta=1 .
\end{aligned}
$$

Solutions of equations (18) and (19) subject to the boundary conditions (21) and (22) are

$$
\begin{aligned}
q(\eta) & =\frac{G_{r}}{r^{2}}\left[\frac { 1 } { 2 P } \left\{\left(\frac{2}{r^{2}} \sin \alpha+\frac{1}{6} F \cos \alpha+\frac{1}{3} \sin \alpha\right) \frac{r \sinh r}{1-\cosh r}\right.\right. \\
& \left.+\left(\sin \alpha+\frac{1}{3} F \cos \alpha+\frac{4}{r^{2}}\right)\right\}\left\{\frac{\sinh r(1-\eta)}{\sinh r}+\frac{\sinh r \eta}{\sinh r}-1\right\}
\end{aligned}
$$

$$
\begin{aligned}
& +\frac{2}{r^{2}}\{\sin \alpha-(1-2 \eta) F \cos \alpha\} \\
& +\left\{(1-\eta)^{2} \sin \alpha-\left(\eta^{2}-\frac{2}{3} \eta^{3}-\frac{1}{3}\right) F \cos \alpha\right\} \\
& -\frac{2}{r^{2}}\left\{\begin{array}{l}
(\sin \alpha-F \cos \alpha) \frac{\sinh r(1-\eta)}{\sinh r} \\
+(\sin \alpha+F \cos \alpha) \frac{\sinh r \eta}{\sinh r}
\end{array}\right\} \\
& \left.-\left(\sin \alpha+\frac{1}{3} F \cos \alpha\right) \frac{\sinh r(1-\eta)}{\sinh r}\right]
\end{aligned}
$$

and

$$
\begin{aligned}
b(\eta) & =\frac{G_{r}}{r^{2}(1+i m)}\left[\left\{\sin \alpha+\frac{1}{3} F \cos \alpha+\frac{2}{r^{2}}(\sin \alpha-F \cos \alpha)\right\}\right. \\
& \times \frac{1-\cosh r(1-\eta)}{r \sinh r} \\
& +\frac{2}{r^{2}}(\sin \alpha+F \cos \alpha) \frac{\cosh r \eta-\cosh r}{r \sinh r}+\frac{1}{3}\left\{(1-\eta)^{3} \sin \alpha\right. \\
& \left.+\left(\eta^{3}-\frac{1}{2} \eta^{4}-\eta\right) F \cos \alpha\right\}-\frac{2}{r^{2}}\{\eta \sin \alpha-\eta(1-\eta) F \cos \alpha\} \\
+ & \frac{1}{6} F \cos \alpha+\frac{2}{r^{2}} \sin \alpha \\
& +\frac{(1-Q)(\eta-1)+\frac{Q}{r \sinh r}\left\{\begin{array}{l}
\cosh r \eta-\cosh r \\
+
\end{array}(1-\eta)\right\}}{1-Q+2 Q \frac{\cosh r-1}{r \sinh r}} \\
& \times\left\{\left(\sin \alpha+\frac{1}{3} F \cos \alpha+\frac{2}{r^{2}}(\sin \alpha-F \cos \alpha)\right) \frac{1-\cosh r}{r \sinh r}\right. \\
& +\frac{2}{r^{2}}(\sin \alpha+F \cos \alpha) \frac{1-\cosh r}{r \sinh r} \\
& \left.\left.+\frac{1}{6} F \cos \alpha+\frac{2}{r^{2}} \sin \alpha+\frac{1}{3} \sin \alpha\right\}\right]
\end{aligned}
$$

where

$$
\begin{aligned}
P=1 & +\frac{1}{2}\left(\frac{1}{Q}-1\right) \frac{r \sinh r}{1-\cosh r}, Q=\frac{M^{2}}{r^{2}(1+i m)}, \quad r=\alpha_{1}-i \beta_{1}, \\
\alpha_{1}, \beta_{1} & =\frac{1}{\sqrt{2}}\left[\left\{\left(\frac{1}{1+m^{2}}+\frac{1}{D a}\right)^{2}+\left(\frac{m M^{2}}{1+m^{2}}-2 K^{2}\right)^{2}\right\}^{\frac{1}{2}}\right. \\
& \left. \pm\left(\frac{1}{1+m^{2}}+\frac{1}{D a}\right)\right]^{\frac{1}{2}} .
\end{aligned}
$$

It is observed from equations (23) and (24) that both the velocity fields and the induced magnetic fields depend on the Grashof number $G r$. In the absence of magnetic field $\left(M^{2}=0\right.$ ) and Hall currents ( $m=0)$, the equation (23) is identical with the equation (14) of Jana et al.[26]. In the absence of rotation ( $\left.K^{2}=0\right)$, angle of inclination $(\alpha=0)$ and porosity of the medium ( $D a=\infty)$, equations (23) and (24) are identical with equations (15) and (16) of Das et al. [29]. In the absence of rotation $\left(K^{2}=0\right)$, angle of inclination $(\alpha=0)$, porosity of the medium $(D a=\infty)$ and Hall currents $(m=0)$ equations (23) and (24) are identical with equations (16) and (17) of Ghosh et al. [25]. 


\section{RESULTS AND DISCUSSION}

We have presented the non-dimensional velocity components and magnetic field components for several values of magnetic parameter $M^{2}$, rotation parameter $K^{2}$, Hall parameter $m$, Grashof number $G r$, Darcy number $D a$ and angle of inclination $\alpha$ against $\eta$ in Figs.2-13. It is seen from Fig.2 that both the primary velocity $u_{1}$ and the secondary velocity $w_{1}$ decrease with an increase in magnetic parameter $M^{2}$. This indicates that the application of the transverse magnetic field plays the role of a resistive type force (Lorentz force) on the flow similar to a drag force (that acts in the opposite direction of the fluid motion), which tends to resist the flow thereby reducing the velocity components. Thus, a magnetic field can be used to control the motion of an electrically conducting fluid. Figs. 3 and 4 show that the primary velocity $u_{1}$ decreases whereas the secondary velocity $w_{1}$ increases with an increase in either rotation parameter $K^{2}$ or Hall parameter $m$. It is found from Figs.5-7 that both the primary velocity $u_{1}$ and the secondary velocity $w_{1}$ increase with an increase in either Grashof number $\mathrm{Gr}$ or Darcy number $\mathrm{Da}$ or angle of inclination $\alpha$. Physically this is due to the fact that an increase in Grashof number $\mathrm{Gr}$ means more heating and less density. Darcy number is the measurement of the porosity of the medium. As the porosity of the medium increases, the value of $D a$ increases. For large porosity of the medium fluid gets more space to flow as a consequence its velocity increases. It is seen from Fig. 8 that the induced magnetic field component $b_{x}$ increases in the region $0 \leq \eta \leq 0.48$ and decreases in the region $0.48<\eta \leq 1.0$ with an increase in magnetic parameter $M^{2}$. On the other hand, $b_{z}$ decreases in the region $0 \leq \eta \leq 0.22$ and increases in the region $0.22<\eta \leq 1.0$ with an increase in magnetic parameter $M^{2}$. Fig.9 shows that the induced magnetic field component $b_{x}$ decreases in the region $0 \leq \eta \leq 0.46$ and increases in the region $0.46<\eta \leq 1.0$ with an increase in rotation parameter $K^{2}$. On the other hand, $b_{z}$ first increases and then decreases with an increase in rotation parameter $K^{2}$. It is found from Fig.10 that the induced magnetic field component $b_{x}$ increases with an increase in Hall parameter $m$. On the other hand, $b_{z}$ decreases in the region $0 \leq \eta \leq 0.26$ and increases in the region $0.26<\eta \leq 1.0$ with an increase in Hall parameter $m$. It is observed from Fig.11 that $b_{x}$ increases in the region $0 \leq \eta \leq 0.2$ and decreases in the region $0.2<\eta \leq 1.0$ with an increase in Grashof number $G r$ while the induced magnetic field component $b_{z}$ increases with an increase in Grashof number $G r$. It is seen from Fig.12 that the induced magnetic field component $b_{x}$ increases in the region $0 \leq \eta \leq 0.32$ and decreases in the region $0.32<\eta \leq 1.0$ while the induced magnetic field component $b_{z}$ increases in the region $0 \leq \eta \leq 0.49$ and decreases in the region $0.49<\eta \leq 1.0$ with an increase in Darcy number $D a$. Fig.13 shows that the induced magnetic field component $b_{x}$ increases in the region $0 \leq \eta \leq 0.16$ and decreases in the region $0.16<\eta \leq 1.0$ with an increase in angle of inclination $\alpha$. On the other hand, $b_{z}$ increases with an increase in angle of inclination $\alpha$. It is observed from Figs.2-13 that the velocity components and the induced magnetic field components become negative away from the wall $\eta=0$ which indicates that there occurs a reverse flow at that region. Physically this is due to the fact that there is a vertical component of the motion of the fluid in the upward direction which is against the gravitational field.

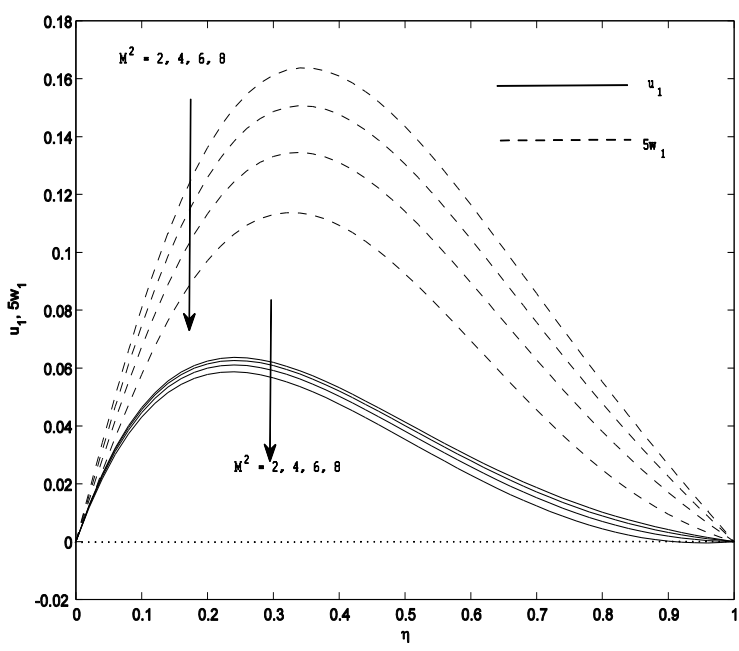

Fig.2: Velocity components $u_{1}$ and $w_{1}$ for $M^{2}$ when $m=0.5, G r=5, K^{2}=10, \alpha=60^{\circ}$ and $D a=0.05$

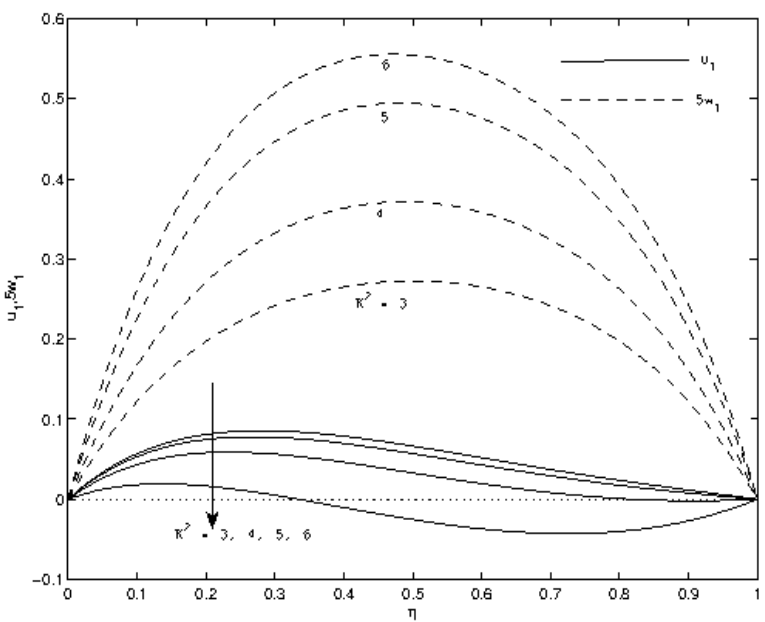

Fig.3: Velocity components $u_{1}$ and $w_{1}$ for $K^{2}$ when $m=0.5, G r=5, M^{2}=10, \alpha=60^{\circ}$ and $D a=0.05$

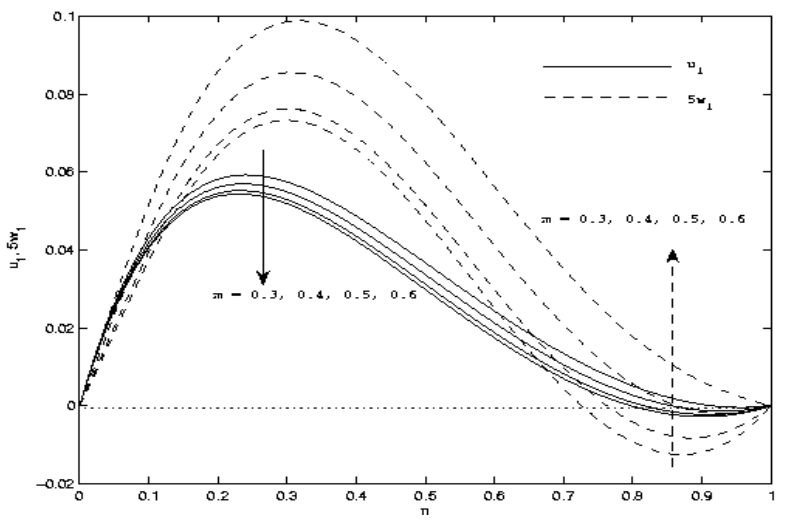

Fig.4: Velocity components $u_{1}$ and $w_{1}$ for $m$ when $M^{2}=10, G r=5, K^{2}=10, \alpha=60^{\circ}$ and $D a=0.05$ 


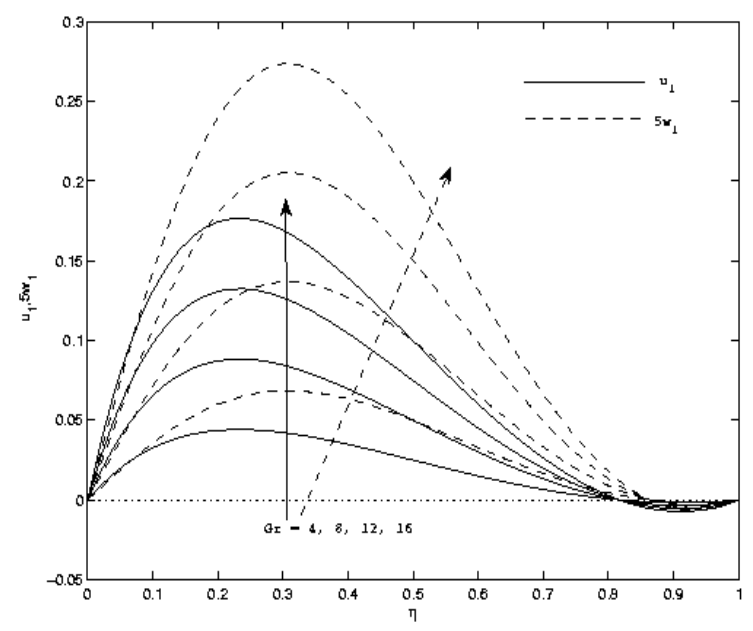

Fig.5: Velocity components $u_{1}$ and $w_{1}$ for $G r$ when $M^{2}=10, m=0.5, K^{2}=10, \alpha=60^{\circ}$ and $D a=0.05$

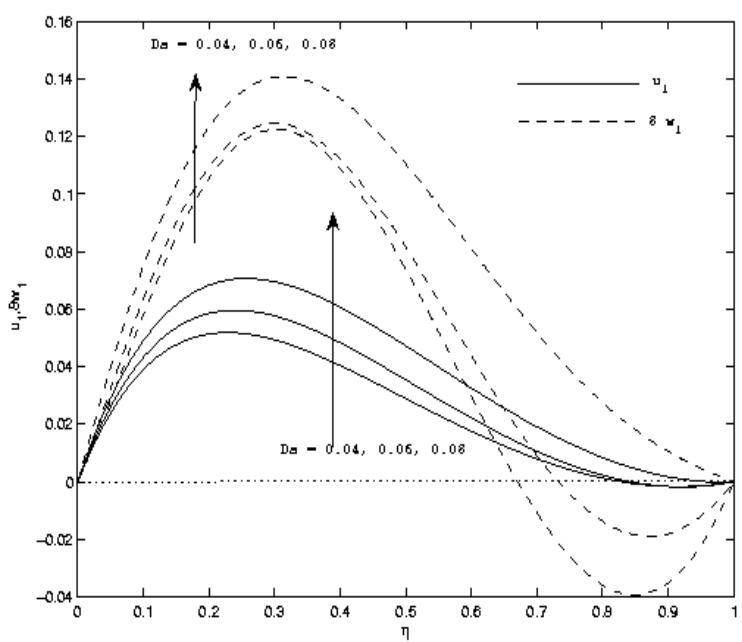

Fig.6: Velocity components $u_{1}$ and $w_{1}$ for $D a$ when $M^{2}=10, G r=5, K^{2}=10, \alpha=60^{\circ}$ and $m=0.5$

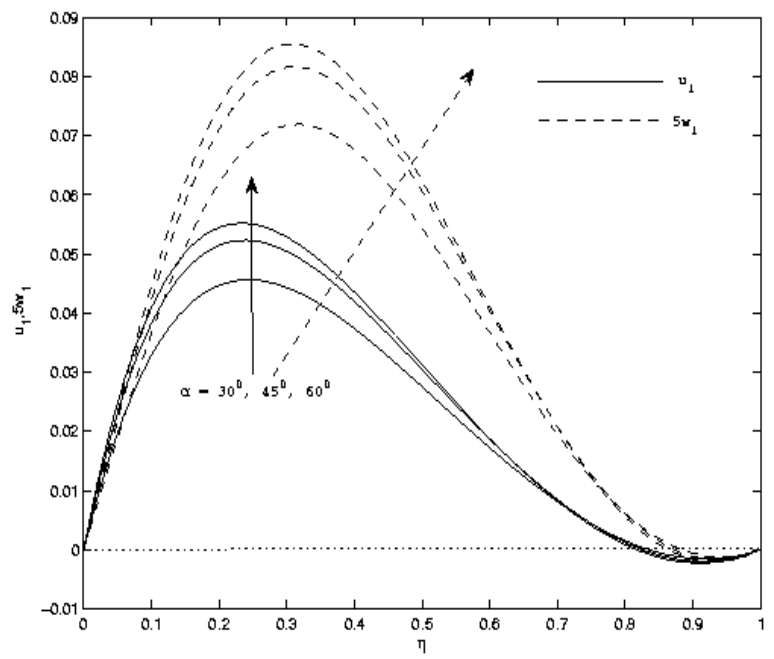

Fig.7: Velocity components $u_{1}$ and $w_{1}$ for $\alpha$ when $M^{2}=10, G r=5, K^{2}=10, m=0.5$ and $D a=0.05$

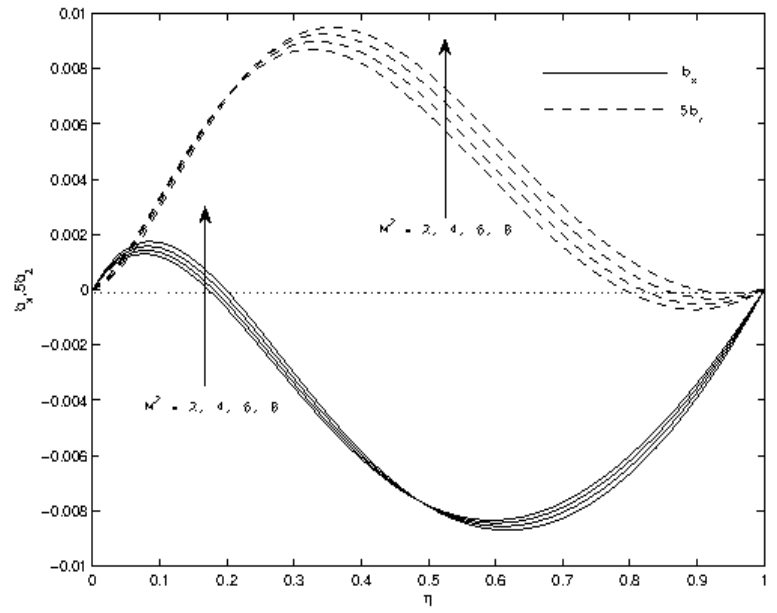

Fig.8: Induced magnetic field components $b_{x}$ and $b_{z}$ for $M^{2}$ when $\alpha=60^{\circ}, G r=5, K^{2}=10, m=0.5$ and $D a=0.05$

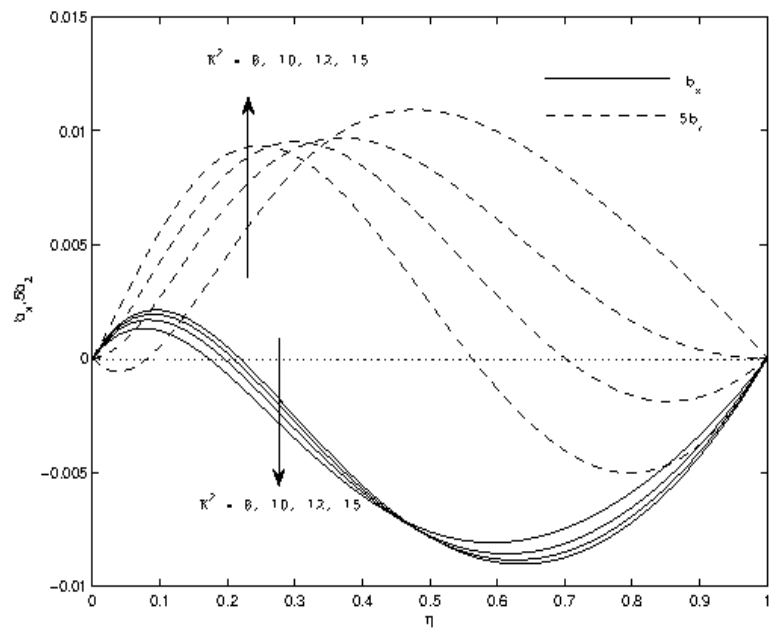

Fig.9: Induced magnetic field components $b_{x}$ and $b_{z}$ for $K^{2}$ when $\alpha=60^{\circ}, G r=5, M^{2}=10, m=0.5$ and $D a=0.05$

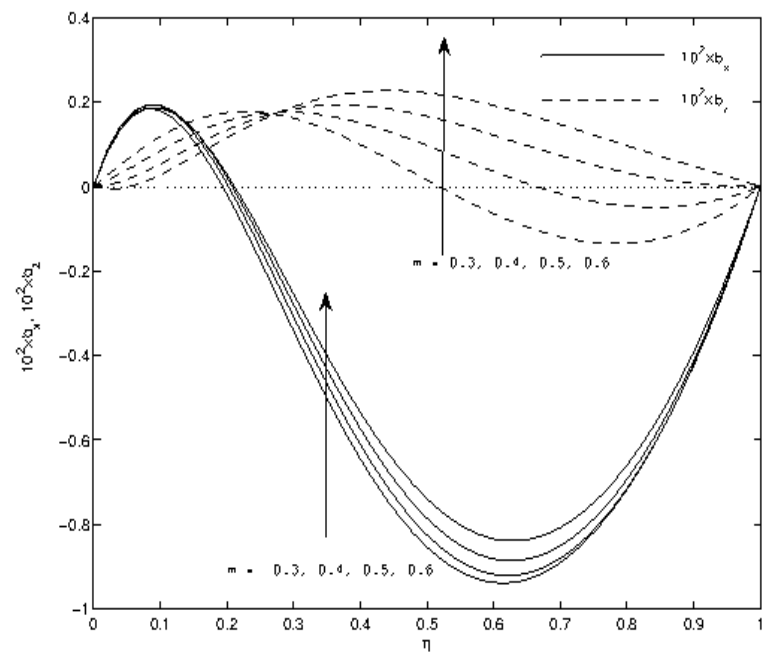

Fig.10: Induced magnetic field components $b_{x}$ and $b_{z}$ for $m$ when $\alpha=60^{\circ}, G r=5, M^{2}=10, K^{2}=10$ and $D a=0.05$ 


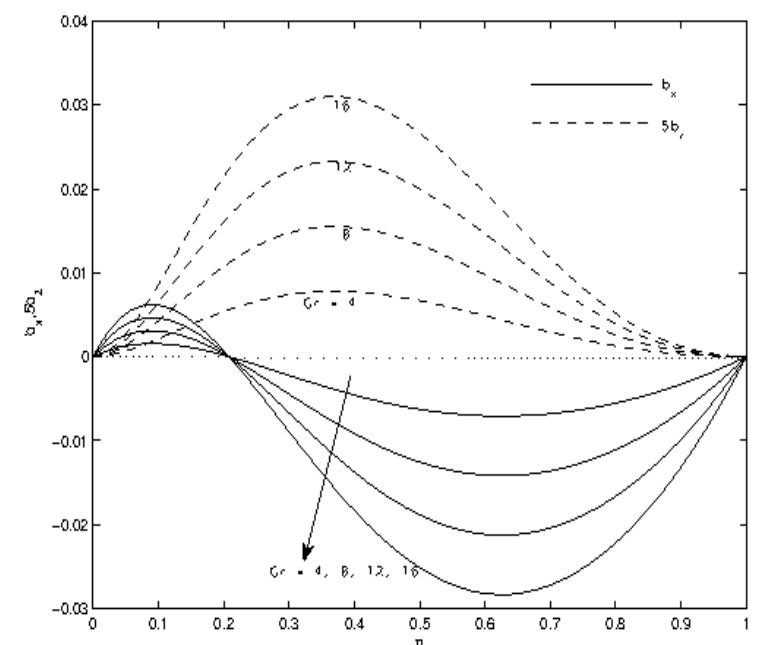

Fig.11: Induced magnetic field components $b_{x}$ and $b_{z}$ for

$G r$ when $\alpha=60^{\circ}, M^{2}=10, K^{2}=10, m=0.5$ and $D a=0.05$

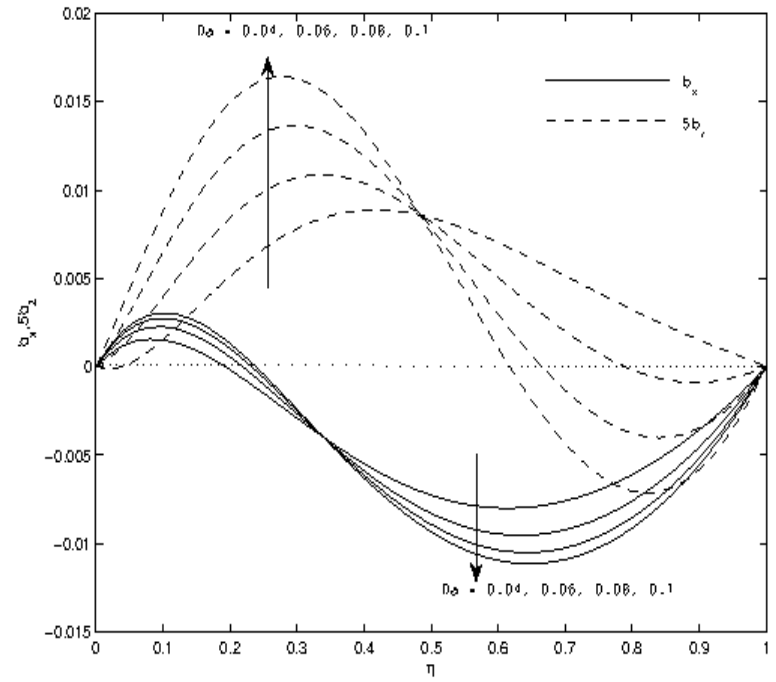

Fig.12: Induced magnetic field components $b_{x}$ and $b_{z}$ for $D a$ when $\alpha=60^{\circ}, G r=5, M^{2}=10, m=0.5$ and $K^{2}=10$

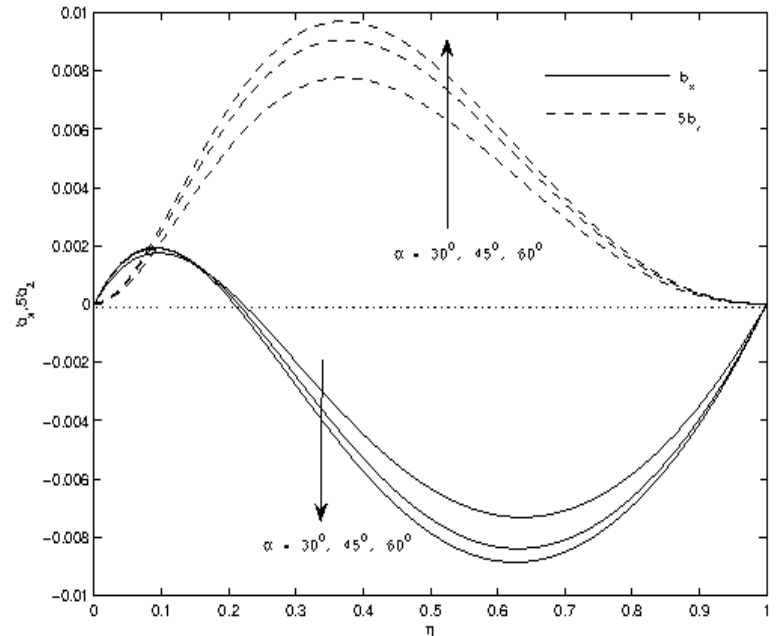

Fig.13: Induced magnetic field components $b_{x}$ and $b_{z}$ for $\alpha$ when $M^{2}=10, G r=5, M^{2}=10, m=0.5$ and $D a=0.05$

The non-dimensional shear stresses at the plate $\eta=0$ due to the primary and secondary flows are

$$
\begin{aligned}
& \tau_{x}+i \tau_{z} \\
& \quad=\frac{G r}{r^{2}}\left[\left\{\frac{1}{P}\left(\frac{r^{2}}{6}+1\right)-2\right\} \sin \alpha+\left(\frac{r^{2}}{12 P}+\frac{4}{r^{2}}\right) F \cos \alpha\right. \\
& +\left(\frac{1}{P}-1\right) \frac{2(1-\cosh r)}{r \sinh r} \sin \alpha-\frac{2(1+\cosh r)}{r \sinh r} F \cos \alpha \\
& \left.+\frac{r}{\sinh r}\left(\sin \alpha+\frac{F \cos \alpha}{3}\right)\left\{\cosh r+\frac{1}{2 P}(1-\cosh r)\right\}\right],
\end{aligned}
$$

where $r$ and $P$ are given by (29). On separating into a real and imaginary parts one can easily obtain the shear stress components $\tau_{x}$ and $\tau_{z}$ from the equation (30).

Numerical results of the shear stresses $\tau_{x}$ and $\tau_{z}$ at the plate $\eta=0$ are presented in Tables 1 and 2 for several values of Hall parameter $m$, angle of inclination $\alpha$, magnetic parameter $M^{2}$ and Darcy number $D a$. Table 1 shows that the shear stress $\tau_{x}$ at the plate due to the primary flow increases with an increase in either angle of inclination $\alpha$ or Darcy number $D a$ whereas it decreases with an increase in magnetic parameter $M^{2}$. It is observed from Table 2 that the shear stress $\tau_{z}$ at the plate due to the secondary flow increases with an increase in either angle of inclination $\alpha$ or Darcy number $D a$ or Hall parameter $m$ whereas it decreases with an increase in magnetic parameter $M^{2}$.

Table 1. Shear stress $\tau_{x}$ at the plate $\eta=0$ when $G r=5$ and $K^{2}=10$

\begin{tabular}{|c|c|c|c|c|c|c|c|c|c|}
\hline & \multicolumn{3}{|c|}{$\alpha$} & \multicolumn{3}{c|}{$M^{2}$} & \multicolumn{3}{c|}{$D a$} \\
\hline$m$ & $30^{0}$ & $45^{0}$ & $60^{0}$ & 1 & 2 & 3 & 0.01 & 0.02 & 0.03 \\
\hline 0.3 & 0.49634 & 0.58357 & 0.62765 & 0.68179 & 0.67721 & 0.67219 & 0.41470 & 0.51350 & 0.56035 \\
0.4 & 0.48272 & 0.56828 & 0.61180 & 0.68234 & 0.67784 & 0.67270 & 0.41575 & 0.51555 & 0.56035 \\
0.5 & 0.47316 & 0.55756 & 0.60069 & 0.68296 & 0.67865 & 0.67357 & 0.41696 & 0.51846 & 0.56317 \\
0.6 & 0.46959 & 0.55359 & 0.59660 & 0.68363 & 0.67958 & 0.67469 & 0.41820 & 0.52177 & 0.56786 \\
\hline
\end{tabular}


Table 2. Shear stress $\tau_{z}$ at the plate $\eta=0$ when $G r=5$ and $K^{2}=10$

\begin{tabular}{|c|c|c|c|c|c|c|c|c|c|}
\hline & \multicolumn{3}{|c|}{$\alpha$} & \multicolumn{3}{c|}{$M^{2}$} & \multicolumn{3}{c|}{$D a$} \\
\hline$m$ & $30^{0}$ & $45^{0}$ & $60^{0}$ & 1 & 2 & 3 & 0.01 & 0.02 & 0.03 \\
\hline 0.3 & 0.55203 & 0.64697 & 0.69414 & 1.77753 & 1.71032 & 1.63506 & 0.31191 & 0.63100 & 0.78609 \\
0.4 & 0.61809 & 0.72075 & 0.77030 & 1.78407 & 1.72081 & 1.65073 & 0.31535 & 0.66033 & 0.86265 \\
0.5 & 0.74486 & 0.86308 & 0.91788 & 1.79166 & 1.73314 & 1.66935 & 0.31828 & 0.68595 & 0.93762 \\
0.6 & 0.90131 & 1.03897 & 1.10044 & 1.79976 & 1.74628 & 1.68915 & 0.32064 & 0.70647 & 1.00159 \\
\hline
\end{tabular}

The bulk temperature in the boundary layer flow is given by

$\theta_{x}+i \theta_{z}=\frac{\int_{0}^{1} q \theta d \eta}{\int_{0}^{1} q d \eta}$

$=\frac{1}{S}\left[\frac{1}{r \sinh r}\left\{\left(\left(\left(1-\frac{1}{P}\right)\left(1+\frac{4}{r^{2}}\right)-\frac{1}{P}-\frac{1}{P}\left(\frac{1}{2}+\frac{2}{r^{2}}\right)\left(1+\frac{4}{r^{2}}\right)\right)\right.\right.\right.$

$\times(1-\cosh r)+\sin \alpha+3)$

$\left.+\frac{1}{3}\left(\left(\left(1-\frac{1}{P}\right)-\frac{1}{P}-\frac{1}{P}\left(\frac{1}{2}+\frac{2}{r^{2}}\right)\right)(1-\cosh r)+3\right) F \cos \alpha\right\}$

$-\frac{2}{r^{3} \sinh r}\left\{\left(1+\frac{4}{r^{2}}\right)(\cosh r-1) \sin \alpha-(1+\cosh r) F \cos \alpha\right\}$

$+\frac{r \sinh r}{1-\cosh r} \frac{1}{P}\left(\frac{2}{r^{2}}+\frac{1}{3}\right)\left(\frac{1}{r^{2}} \sin \alpha+\frac{1}{12} F \cos \alpha+\frac{1}{6} \sin \alpha\right)$

$+\left\{\frac{4}{r^{2}}+\frac{14}{3 r^{2}}-\frac{2}{r^{2}} \cosh r+\frac{1}{5}-\frac{1}{P}\left(\frac{40}{3 r^{2}}+\frac{8}{r^{4}}-\frac{1}{3}\right)\right\} \sin \alpha$

$\left.+\left\{\frac{12}{r^{4}}+\frac{1}{3 r^{2}}+\frac{13}{45}-\frac{1}{P}\left(\frac{10}{3 r^{2}}+\frac{65}{36}\right)\right\} F \cos \alpha\right]$,

where

$$
\begin{aligned}
S & =\frac{1}{P}\left\{\left(\frac{1}{r^{2}} \sin \alpha+\frac{1}{12} F \cos \alpha+\frac{1}{6} \sin \alpha\right) \frac{r \sinh r}{\cosh r-1}\right. \\
& \left.-\frac{1}{2}\left(\sin \alpha+\frac{1}{3} F \cos \alpha+\frac{4}{r^{2}} \sin \alpha\right)\right\} \\
& +\left(1-\frac{1}{P}\right)\left\{\left(\sin \alpha+\frac{1}{3} F \cos \alpha+\frac{4}{r^{2}} \sin \alpha\right) \frac{1-\cosh r}{r \sinh r}\right. \\
& \left.+\left(\frac{2}{r^{2}}+\frac{1}{3}\right) \sin \alpha+\frac{1}{6} F \cos \alpha\right\} .
\end{aligned}
$$

where $r$ and $P$ are given by (29).

It is interesting to note from the above equation (27) that the bulk temperature in the boundary layer flow does not depend on the Grashof number $\mathrm{Gr}$.

Numerical results of the bulk temperature in the boundary layer flow are presented in Table-3 for several values of Hall parameter $m$ and squared-Hartmann number $M^{2}$. Table-3 shows that the magnitude of the bulk temperature $\theta_{x}$ in the $x$ direction as well as the bulk temperature $\theta_{z}$ in the $z$-direction increase with an increase in magnetic parameter $M^{2}$ whereas they decrease with an increase in Hall parameter $m$.

Table 3. Bulk temperatures $-\theta_{x}$ and $\theta_{z}$ at the plate $\eta=0$

\begin{tabular}{|c|c|c|c|c|c|c|c|c|}
\hline & \multicolumn{4}{|c|}{$\theta_{x}$} & \multicolumn{4}{c|}{$\theta_{z}$} \\
\hline$m \backslash M^{2}$ & 1 & 2 & 3 & 4 & 1 & 2 & 3 & 4 \\
\hline 0.3 & 0.94747 & 1.00929 & 1.08004 & 1.16219 & 1.68450 & 1.70571 & 1.72844 & 1.75198 \\
0.4 & 0.94627 & 1.00921 & 1.08008 & 1.16137 & 1.67676 & 1.69624 & 1.71875 & 1.74452 \\
0.5 & 0.94342 & 1.00600 & 1.07511 & 1.15289 & 1.66844 & 1.68588 & 1.70739 & 1.73392 \\
0.6 & 0.93940 & 1.00057 & 1.06674 & 1.13951 & 1.66003 & 1.67525 & 1.69508 & 1.72091 \\
\hline
\end{tabular}

\section{ENTROPY GENERATION}

The entropy generation in a fluid is caused by exchange of momentum and energy within the fluid and at the boundaries. This causes continuous entropy generation. One portion of this entropy production is due to heat transfer in the direction of finite temperature gradients. Finite temperature difference heat transfer irreversibly is common in all types of thermal engineering applications. Another portion of the entropy production arises due to fluid friction irreversibility. The volumetric rate of entropy generation in the presence of a magnetic field is given by

$$
\begin{aligned}
E_{G} & =\frac{k^{\prime}}{T_{\infty}^{2}}\left(\frac{\partial T}{\partial y}\right)^{2}+\frac{\mu}{T_{\infty}}\left[\left(\frac{\partial u}{\partial y}\right)^{2}+\left(\frac{\partial w}{\partial y}\right)^{2}\right]+\frac{1}{\sigma T_{\infty}}\left[\left(\frac{\partial B_{x}}{\partial y}\right)^{2}+\left(\frac{\partial B_{z}}{\partial y}\right)^{2}\right] \\
& +\frac{\mu^{\prime}}{k^{*} T_{\infty}}\left(u^{2}+w^{2}\right) .
\end{aligned}
$$

The first term on the right-hand side of (29) is the local entropy generation due to heat transfer across a finite temperature difference, the second term is the local entropy generation due to viscous dissipation, the third term is the local entropy generation due to the effect of the magnetic field and fourth term is the local entropy generation due to fluid friction.

The dimensionless entropy generation number may be defined by the following relationship:

$$
N_{S}=\frac{T_{\infty}^{2} \delta^{2} E_{G}}{\left(T_{w}-T_{\infty}\right)^{2}} .
$$

In terms of the dimensionless velocity and temperature, the entropy generation number becomes

$$
\begin{aligned}
N_{S} & =\left(\frac{\partial \theta}{\partial \eta}\right)^{2}+\frac{B r}{\Omega}\left[\left\{\left(\frac{\partial u_{1}}{\partial \eta}\right)^{2}+\left(\frac{\partial w_{1}}{\partial \eta}\right)^{2}\right\}+M^{2}\left\{\left(\frac{\partial b_{x}}{\partial \eta}\right)^{2}+\left(\frac{\partial b_{z}}{\partial \eta}\right)^{2}\right\}\right] \\
& +\frac{B r^{*}}{\Omega D a}\left(u_{1}^{2}+w_{1}^{2}\right)
\end{aligned}
$$


where $B r=\frac{\mu_{e} v^{2}}{k^{\prime}\left(T_{w}-T_{\infty}\right) \delta^{2}} \quad$ is the Brinkmann number, $B r^{*}=\frac{\mu v^{2}}{k^{*}\left(T_{w}-T_{\infty}\right) \delta^{2}}$ the modified Brinkmann number and $\Omega=\frac{T_{w}-T_{\infty}}{T_{\infty}}$ dimensionless temperature difference.

The entropy in a system is associated with the presence of irreversibility. We have to notice that the contribution of the heat transfer entropy generation to the overall entropy generation rate is needed in many engineering applications. The Bejan number $B e$ is an alternative irreversibility distribution parameter and it represents the ratio between the heat transfer irreversibility $N_{h}$ and the total irreversibility $N_{S}$. It is defined by

$$
B e=\frac{N_{h}}{N_{S}} .
$$

The Bejan number takes the values between 0 and 1 (see Cimpean et al. [34]). The value of $B e=1$ is the limit at which the heat transfer irreversibility dominates, $B e=0$ is the opposite limit at which the irreversibility is dominated by fluid friction effects and $B e=0.5$ is the case in which the heat transfer and fluid friction entropy production rates are equal (see Varol et al. [35, 36]). Further, the behavior of the Bejan number is studied for the optimum values of the parameters at which the entropy generation takes its minimum.

The influences of the different governing parameters on entropy generation are presented in Figs. 14-27. It is seen from Figs.1419 that the entropy generation number $N_{S}$ increases with an increase in either magnetic parameter $M^{2}$ or Hall parameter $m$ or Grashof number $\mathrm{Gr}$ or porosity parameter $\mathrm{Da}$ or $B r \Omega^{-1}$ or $B r^{*} \Omega^{-1}$. An increase in the magnetic field intensity causes a general increase of the entropy generation number. Fig.20 shows that the entropy generation number $N_{S}$ decreases with an increase in rotation parameter $K^{2}$. Figs.21-22 shows the Bejan number $B e$ increases with an increase in either magnetic parameter $M^{2}$ or rotation parameter $K^{2}$. The effect of Hall parameter $m$ on the Bejan number $B e$ is shown in Fig.23. The figure reveals an increase in the Bejan number $B e$ as $m$ increases. It is observed from Figs.24-27 that the Bejan number $B e$ decreases with an increase in either Grashof number $G r$ or porosity parameter $\mathrm{Da}$ or $\mathrm{Br} \Omega^{-1}$ or $\mathrm{Br}^{*} \Omega^{-1}$. The group parameter is an important dimensionless number for irreversibility analysis. It determines the relative importance of viscous effects to that of temperature gradient entropy generation. An increase in the values of the group parameter due to the combined effects of viscous heating and temperature difference yields a higher entropy generation rate.

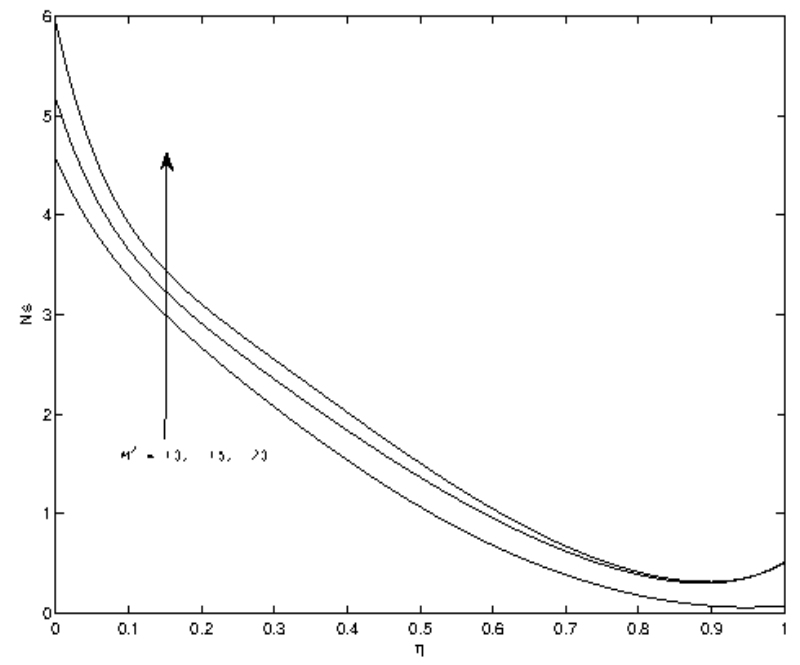

Fig.14: $N_{S}$ for different $M^{2}$ when $M^{2}=10, G r=5$, $M^{2}=10, m=0.5$ and $D a=0.05$

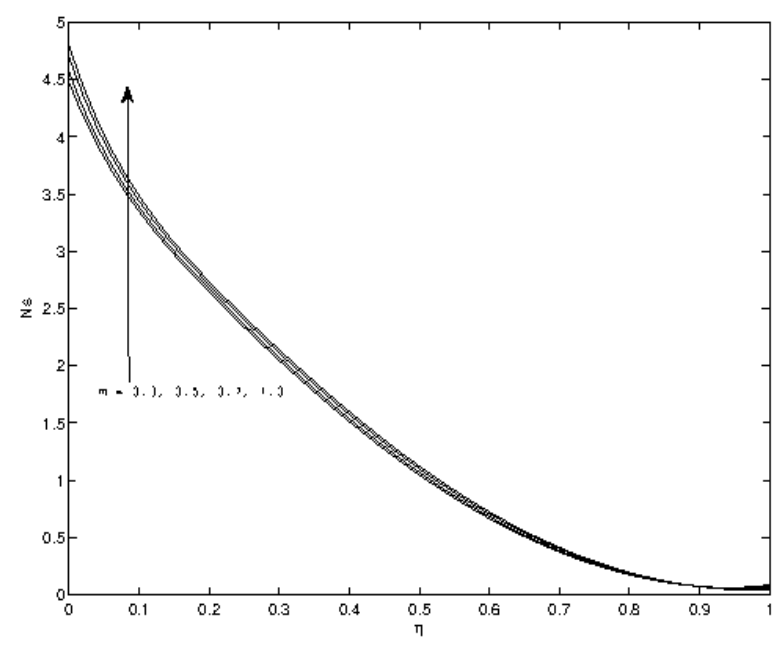

Fig.15: $N_{S}$ for different $m$ when $M^{2}=10, G r=5$, $M^{2}=10, m=0.5$ and $D a=0.05$

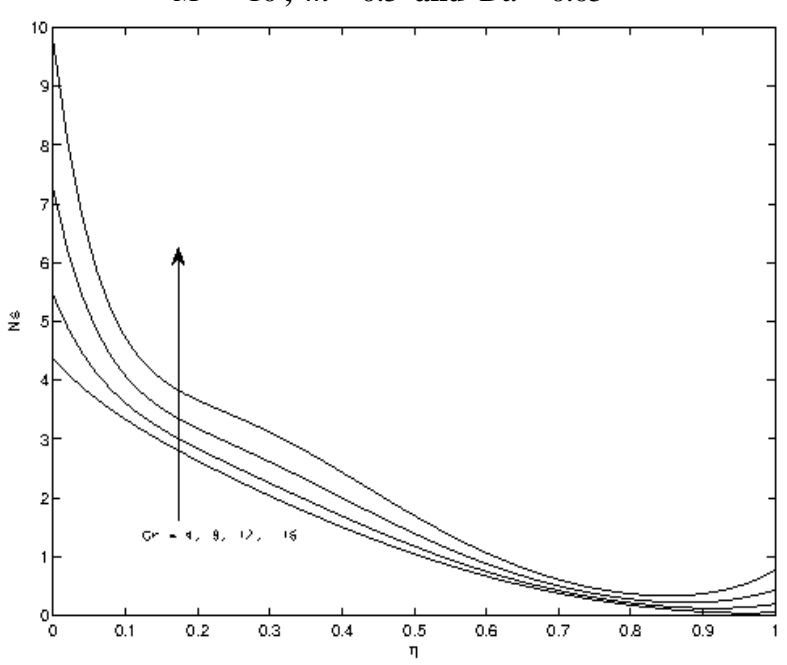

Fig.16: $N_{S}$ for different $G r$ when $M^{2}=10, G r=5$, $M^{2}=10, m=0.5$ and $D a=0.05$ 


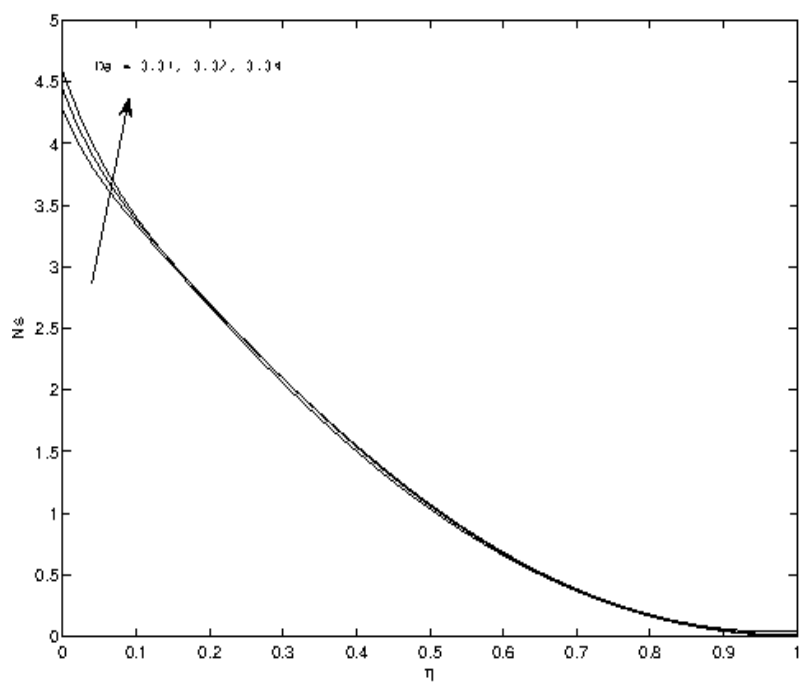

Fig.17: $N_{S}$ for different $D a$ when $M^{2}=10, G r=5$, $M^{2}=10, m=0.5$ and $D a=0.05$

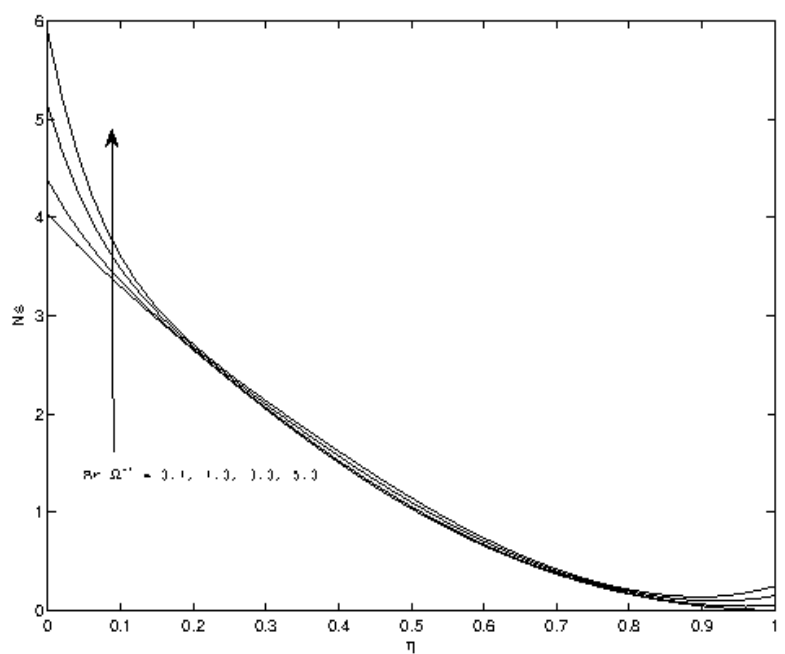

Fig.18: $N_{S}$ for different $B r \Omega^{-1}$ when $M^{2}=10, G r=5$, $m=0.5$ and $D a=0.05$

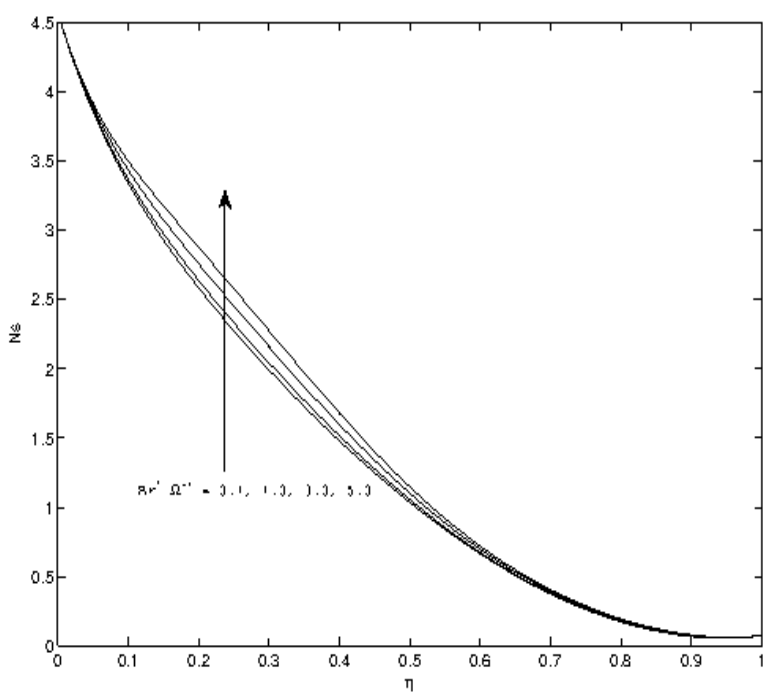

Fig.19: $N_{S}$ for different $B r^{*} \Omega^{-1}$ when $M^{2}=10, G r=5$, $M^{2}=10, m=0.5$ and $D a=0.05$

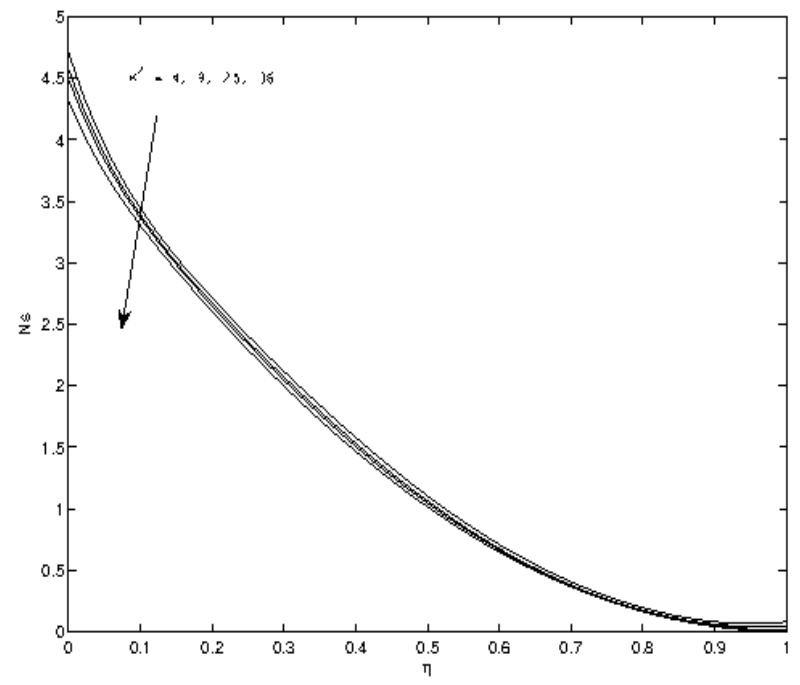

Fig.20: $N_{S}$ for different $K^{2}$ when $M^{2}=10, G r=5$, $M^{2}=10, m=0.5$ and $D a=0.05$

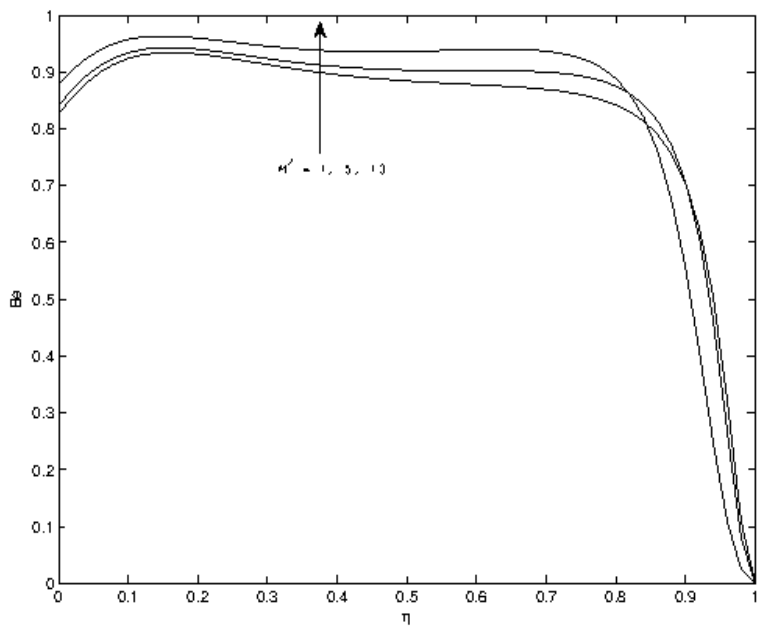

Fig.21: $B e$ for different $M^{2}$ when $M^{2}=10, G r=5$, $M^{2}=10, m=0.5$ and $D a=0.05$

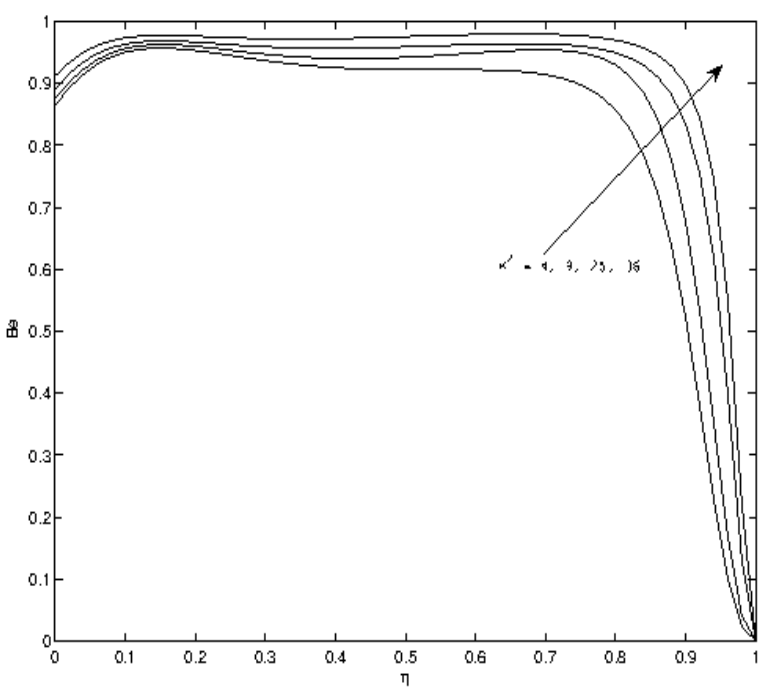

Fig.22: $B e$ for different $K^{2}$ when $M^{2}=10, G r=5$, $M^{2}=10, m=0.5$ and $D a=0.05$ 


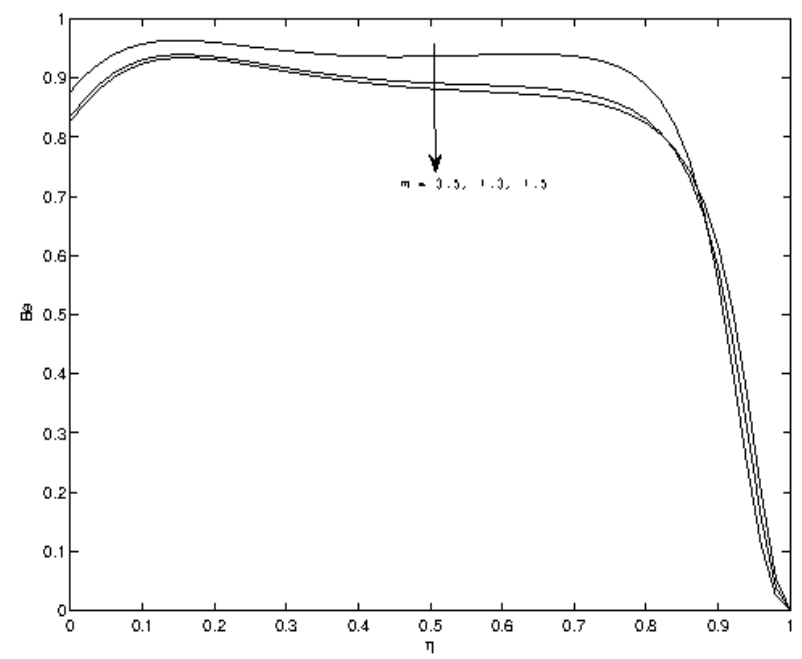

Fig.23: $B e$ for different $m$ when $M^{2}=10, G r=5$, $M^{2}=10, m=0.5$ and $D a=0.05$

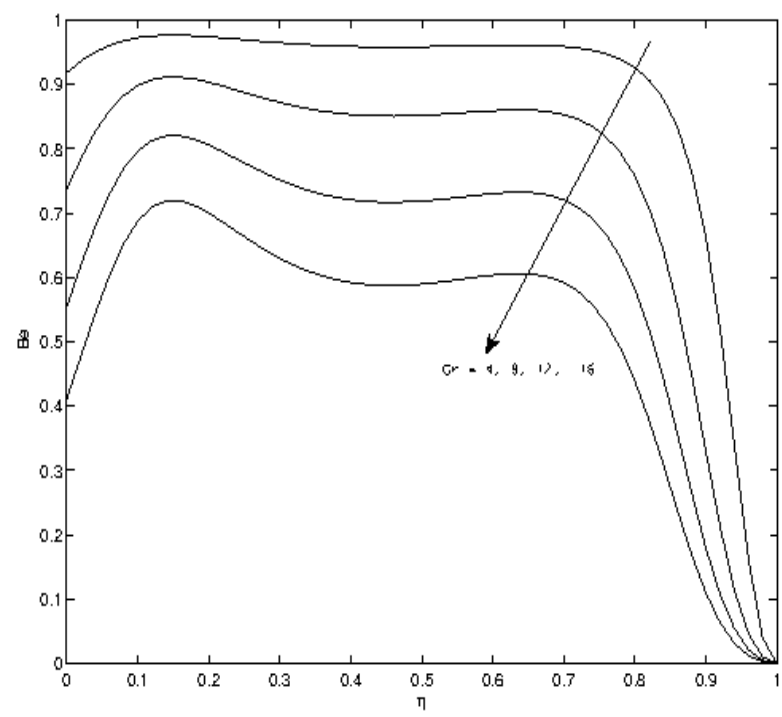

Fig.24: $B e$ for different $G r$ when $M^{2}=10, G r=5$, $M^{2}=10, m=0.5$ and $D a=0.05$

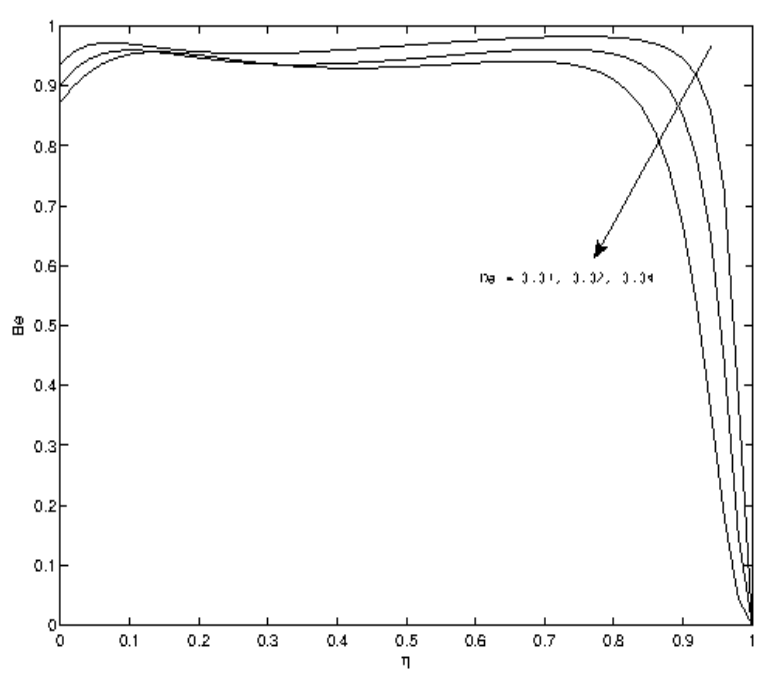

Fig.25: $B e$ for different $D a$ when $M^{2}=10, G r=5$, $M^{2}=10, m=0.5$ and $D a=0.05$

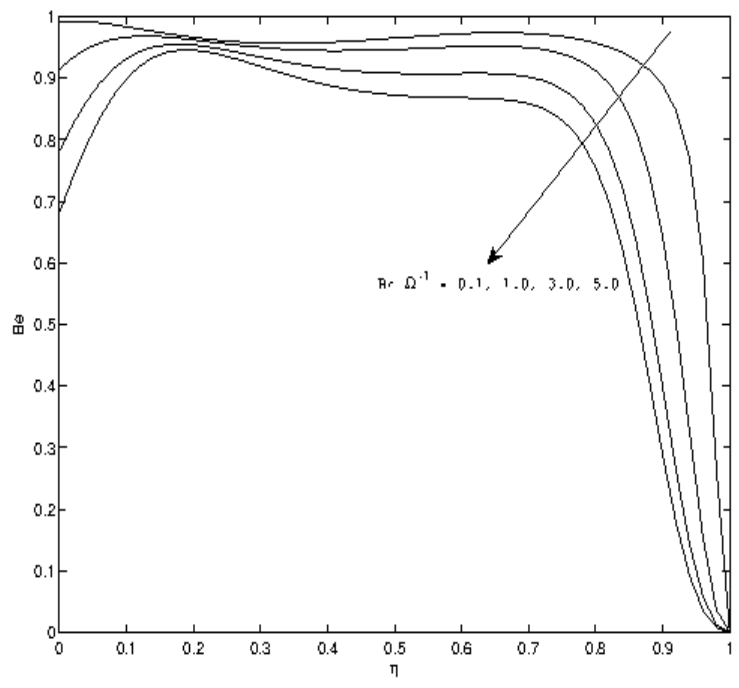

Fig.26: $B e$ for different $B r \Omega^{-1}$ when $M^{2}=10, G r=5$, $M^{2}=10, m=0.5$ and $D a=0.05$

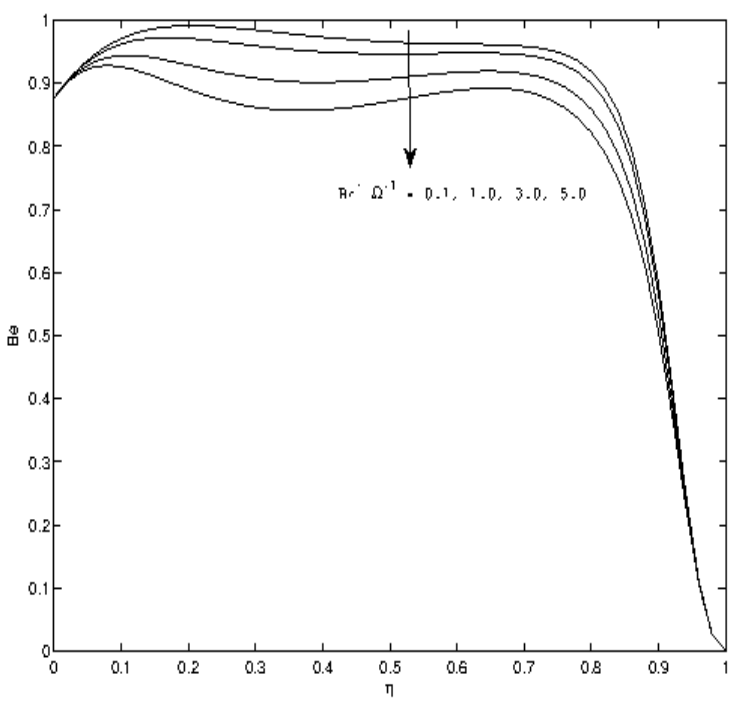

Fig.27: $B e$ for different $B r^{*} \Omega^{-1}$ when $M^{2}=10, G r=5$, $M^{2}=10, m=0.5$ and $D a=0.05$

\section{CONCLUSION}

Entropy generation analysis in a hydromagnetic free convective boundary layer flow past an inclined flat plate embedded in a porous medium in the presence of a uniform transverse magnetic field taking Hall currents into account has been carried out. The magnetic field significantly controls the fluid flow. The buoyancy force as well as the porosity of the porous medium accelerate the fluid velocity components. The induced magnetic field components are radically influenced by the Hall currents. Hall currents reduce the bulk temperature at the plate. It is interesting to note that there is no effect of the buoyancy force on the bulk temperatures. Bejan number enhances as Hall parameter increases. The entropy generation increases with an increase in either magnetic parameter or Hall parameter or Grashof number or porosity parameter. The present study find applications in cooling problems in the industry, to control the boundary layer separations and to reduce the drag etc. 


\section{REFERENCES}

[1] Ghosh, S. K. and Pop, I. (2005). A new approach on MHD natural convection boundary layer flow past a flat plate of finite dimensions, Int. J. Heat Mass Transfer, 42: 587-595.

[2] Katagiri, M. (1969). The effect of Hall currents on the magnetohydrodynamic boundary layer flow past a semiinfinite flat plate, J. Phys. Soc. Jpn, 27: 1051-1059.

[3] Pop, I., Watanabe, Y. (1995). Hall effects on magnetohydrodynamic boundary layer flow over a continuous moving flat plate, Acta Mech., 108:35-47.

[4] Fujii, T., Imura, H. (1972). Natural convection heat transfer from a plate with arbitrary inclination, Int. J. Heat Mass Trans., 15: 755.

[5] Kierkus, W.(1968). An analysis of laminar free convection flow and heat transfer about an inclined isothermal plate, Int. J. Heat Mass Trans., 11: 241-253.

[6] Ganesan, P., Palani, G. (2003). Natural convection effects on impulsively started inclined plate with heat and mass transfer, Int. J. Heat Mass Trans., 39: 277-283.

[7] Moutsoglou, A., Chen, T. S. (1980). Buoyancy effects in the boundary layers on inclined, continous, moving sheets, ASME J. Heat Trans., 102: 371-373.

[8] Lewandowski, W. M. (1991). Natural convection heat transfer from plates of finite dimension, Int. J. Heat Mass Trans., 34: 875-885.

[9] Bejan, A. (1980). Second law analysis in heat transfer, Energy Int. J., 5: 721-732.

[10] Bejan, A. (1994). Entropy generation through heat and fluid flow, Wiley, Canada.

[11] Bejan, A. (1982). Second-law analysis in heat transfer and thermal design, Adv. Heat Transfer, 15: 1-58.

[12] Bejan, A. (1996). Entropy Generation Minimization, CRC Press: New York, USA.

[13] Bejan, A. (1979). A study of entropy generation in fundamental convective heat transfer, J. Heat Transfer, 101: 718-725.

[14] Bejan, A. (1996).Tsatsaronis, G. and Moran, M., Thermal Design and Optimization; Wiley: New York, USA.

[15] Arpaci, V.S. and Selamet, A. (1988). Entropy production in flames, Combust. Flame, 73: 254-259.

[16] Arpaci, V.S. and Selamet, A.(1990). Entropy production in boundary layers, J. Thermophys. Heat Transfer, 4: 404-407.

[17] Arpaci, V.S.(1991). Radiative entropy production-Heat lost to entropy, Adv. Heat Transfer, 21: 239-276.

[18] Arpaci, V.S.(2001). Thermal deformation: From thermodynamics to heat transfer, J. Heat Transfer, 123: 821826.

[19] Arpaci, V.S. and Esmaeeli, A.(2000). Radiative deformation, J. Appl. Phys., 87: 3093-3100.

[20] Magherbi, M., Abbassi, H. and Ben Brahim, A. (2003). Entropy generation at the onset of natural convection, Int. J. Heat Mass Transfer, 46: 3441-3450.
[21] Magherbi, M., Abbassi, H., Hidouri N. and Ben Brahim, A. (2006). Second law analysis in convective heat and mass transfer, Entropy, 8: 1-17.

[22] Abbassi, H., Magherbi, M. and Ben Brahim, A. (2003). Entropy generation in Poiseuille-Benard channel flow, Int. J. Therm. Sci., 42:1081-1088.

[23] Cimpean, D. and Pop I. (2011). A study of entropy generation minimization in an inclined channel, WSEAS Transactions on Heat and Mass Transfer, 6(2): 31-40.

[24] Chen, H. T. and Chen, C. K. (1988). Free convection flow of non-Newtonian fluids along a vertical plate embedded in porous medium, ASME J. Heat Tranfer., 110: 257-260.

[25] Ghosh, S. K., Bég, O. A. and Zueco, J. (2010). Hydromagnetic free convection flow with induced magnetic field effects, Meccanica, 45: 175-185.

[26] Jana, M., Das, S., Maji, S. L., Jana, R. N. and Ghosh, S. K. (2012). Natural convection boundary layer flow past a flat plate of finite dimensions, J. Porous Media, 15(6): 585593.

[27] Ahmed, N. and Kalita, D. (2011). Transient MHD free convection from an infinite vertical Porous plate in a rotating system with mass transfer and Hall current, J. Ener. Heat Mass Transfer, 33: 271- 292.

[28] Ahmed, N., Talukdar, S. (2012). Transient magnetohydrodynamic (MHD) flow of a visco-elastic fluid past an infinite vertical porous plate embedded in a porous medium with Hall current and slip condition in a rotating system, Int. J. Physical Sci., 7(45): 5942-5953.

[29] Das, S., Sarkar, B. C. and Jana, R. N. (2013). Hall effect on MHD free convection boundary layer flow past a vertical flat plate, Meccanica, 48:1387-1398.

[30] Makinde, O. D.(2009). On MHD boundary-layer flow and mass transfer past a vertical plate in a porous medium with constant heat flux. Int. J. Num. Methods for Heat \& Fluid Flow, 19(3/4): 546-554.

[31] Kumar, R. and Chand, K.(2011). Effect of slip conditions and hall current on unsteady MHD flow of a viscoelastic fluid past an infinite vertical porous plate through porous medium, Int. J. Eng. Sci. Tech., 3(4):3124-3123.

[32] Makinde, O. D. (2012). Entropy analysis for MHD boundary layer flow and heat transfer over a flat plate with a convective surface boundary condition, Int. J. Exergy, 10(2): 142-154.

[33] Cowling, T. G. (1957). Magnetohydrodynamics, Interscience, New York.

[34] Cimpean, D., Lungu, N. and Pop, I. (2008). A problem of entropy generation in a channel filled with a porous medium, Creative Math. and Inf., 17: 357-362.

[35] Varol, Y., Oztop, H.F. and Koca, A., Entropy production due to free convection in partially heated isosceles triangular enclosures, Applied Thermal Engineering, 28: 1502-1513.

[36] Varol, Y., Oztop, H.F. and Pop, I. (2008). Numerical analysis of natural convection for a porous rectangular enclosure with sinusoidally varying temperature profile on the bottom wall, Int. Comm. Heat Mass Transfer, 35: 56-64. 